\title{
Comparative Infinite Lottery Logic
}

Matthew W. Parker

\begin{abstract}
As an application of his Material Theory of Induction, Norton (2018; manuscript) argues that the correct inductive logic for a fair infinite lottery, and also for evaluating eternal inflation multiverse models, is radically different from standard probability theory. This is due to a requirement of label independence. It follows, Norton argues, that finite additivity fails, and any two sets of outcomes with the same cardinality and co-cardinality have the same chance. This makes the logic useless for evaluating multiverse models based on self-locating chances, so Norton claims that we should despair of such attempts. However, his negative results depend on a certain reification of chance, consisting in the treatment of inductive support as the value of a function, a value not itself affected by relabeling. Here we define a purely comparative infinite lottery logic, where there are no primitive chances but only a relation of 'at most as likely' and its derivatives. This logic satisfies both label independence and a comparative version of additivity as well as several other desirable properties, and it draws finer distinctions between events than Norton's. Consequently, it yields better advice about choosing between sets of lottery tickets than Norton's, but it does not appear to be any more helpful for evaluating multiverse models. Hence, the limitations of Norton's logic are not entirely due to the failure of additivity, nor to the fact that all infinite, co-infinite sets of outcomes have the same chance, but to a more fundamental problem: We have no wellmotivated way of comparing disjoint infinite sets.
\end{abstract}




\section{Introduction.}

Norton's Material Theory of Induction denies that there is a single, universally applicable logic of inductive inference. Rather, it proposes that the warrant for any inductive inference lies entirely in the background facts of the particular application (manuscript). A striking example is the case of a fair infinite lottery (ibid.; 2018). According to Norton, the inductive logic for such a lottery must satisfy a strong fairness condition called label independence, which renders the logic extremely weak and quite unlike standard (i.e., Kolmogorovian) probability theory.

In order to refer to particular lottery outcomes, we must assign them labels. Norton also assigns to each set $\mathrm{A}$ of possible outcomes a value $\mathrm{Ch}(\mathrm{A})$ that he calls a chance, which is to be understood as a degree of inductive support but is not necessarily a number. The condition required for a lottery to be fair, on Norton's view, is

Label independence: All true statements pertinent to the chances of different outcomes remain true when the labels are arbitrarily permuted (manuscript).

Given label independence, Norton shows that, not only countable additivity, but even

Finite additivity: If $\mathrm{A}$ and $\mathrm{B}$ are disjoint, then $\mathrm{Ch}(\mathrm{A} \cup \mathrm{B})=\mathrm{Ch}(\mathrm{A})+\mathrm{Ch}(\mathrm{B}),{ }^{1}$

\footnotetext{
${ }^{1}$ This definition of finite additivity does not assume that $\mathrm{Ch}$ is real-valued, only that there is a designated operation ' + ' over the range of $\mathrm{Ch}$. There is no such designated operation for Norton's chances, but he shows that, given label independence, $\mathrm{Ch}$ cannot be a real valued, finitely additive probability function, with ordinary addition. (His argument also applies to hyperreals.) We will show here that, whatever sort of values $\mathrm{Ch}$ takes, if it is not constant and label independence holds then there is no operation ' + ' that makes it finitely additive (Proposition 1).
} 
fails. He also shows that, given label independence, any infinite set of outcomes with an infinite complement has the same chance as any other such set. So, for example, if we label the tickets with positive integers, the proposition that the winning number will be even has exactly as much support as the proposition that it will be prime, or that it will be composite. Another property that fails in Norton's logic is

Containment: If $\mathrm{A}$ is a proper subset of $\mathrm{B}$, then the chance of $\mathrm{A}$ is strictly less than that of $\mathrm{B}$, i.e., $\mathrm{A} \subset \mathrm{B} \Rightarrow \mathrm{Ch}(\mathrm{A})<\mathrm{Ch}(\mathrm{B})$.

Containment does not generally hold in standard probability theory, but it has been regarded by some as desirable or plausible, especially by supporters of hyperreal probabilities (taking infinitesimal values, real values, and combinations thereof) and of regularity, the property that no possible event has probability zero. (See Benci et al. 2018 for references and discussion.) But, alas, a chance function that satisfies label independence over an infinite sample space cannot satisfy containment, as we will see.

The infinite lottery may be more than an imaginary game. Norton argues that his infinite lottery logic is also the correct inductive logic for self-location within the infinite multiverse of eternal inflation cosmology. Eternal inflation hypothesizes that our "universe" is just one of infinitely many spatially infinite bubbles of stability in a rapidly expanding background space. According to the Principle of Mediocrity (Vilenkin 1995), we ought to think of ourselves as living in a randomly chosen civilization among the infinitely many civilizations scattered across these worlds. Hence, if a particular model of inflation makes worlds like ours quite typical, then the fact that we do live in such a world incrementally confirms the model, and if it makes worlds like ours atypical, that incrementally disconfirms the model. Norton reasons that if our civilization, or our world, is chosen arbitrarily, then the 
chance of finding ourselves in this or that kind of world must be invariant under a relabeling of worlds. Hence the degree to which the properties of our local world confirm or disconfirm a given model must be calculated by the same strange, weak logic that applies to fair infinite lotteries.

But if this is correct, there is little calculation to do. For any non-trivial property of worlds, in any plausible model of eternal inflation there are infinitely many worlds with that property and infinitely many without it. Hence, on Norton's logic, we are no more likely to find ourselves in one kind of world than another, so the fact that we observe a world like ours does nothing to confirm or disconfirm the model. Nonetheless, Norton argues, this is the correct logic for the domain, as warranted by the background facts. Hence, he concludes, we must despair of confirming or disconfirming eternal inflation models in this way.

I will give a brief exposition of Norton's infinite lottery logic, mention some concerns, and strengthen his proof that additivity fails. However, the main purpose of this note is to show that Norton's negative results depend not only on label independence but also on some tacit assumptions. Norton seems to assume that chances are, in a certain sense, things: They are values of a function "Ch". Because of this, we can say things like "The chance that the outcome is in set $\mathrm{S}$ is $\mathrm{X}^{\prime}$, and therefore label independence seems to force us to assign the same chance $\mathrm{X}$ to any set that is related to $\mathrm{S}$ by a relabeling. Consequently, any two sets with the same cardinality and co-cardinality (the cardinality of the complement in the set of all possible outcomes) have the same chance, and finite additivity fails.

Another tacit assumption involved in this reasoning is the assumption that chances are not things that are themselves modified by a relabeling of outcomes. We might, for example, identify the chance of an outcome set with the equivalence class of all equally supported sets. In that case, if a relabeling (permutation) maps a set $\mathrm{A}$ to $\mathrm{B}$, then it also maps $\mathrm{Ch}(\mathrm{A})=[\mathrm{A}]=$ $\{\mathrm{X}$ : $\mathrm{A}$ and $\mathrm{X}$ are equally supported $\}$ to $\mathrm{Ch}(\mathrm{B})=[\mathrm{B}]=\{\mathrm{X}$ : $\mathrm{B}$ and $\mathrm{X}$ are equally supported $\}$. 
That is, under the relabeling, ' $\mathrm{Ch}(\mathrm{A})=[\mathrm{A}]$ ' becomes ' $\mathrm{Ch}(\mathrm{B})=[\mathrm{B}]$ ', which holds even if $[\mathrm{A}]$ is not equal to $[\mathrm{B}]$. So $\mathrm{Ch}(\mathrm{A})=[\mathrm{A}]$ does not imply that $\mathrm{Ch}(\mathrm{B})=[\mathrm{A}]$, nor, therefore, that $\mathrm{Ch}(\mathrm{A})=\mathrm{Ch}(\mathrm{B})$. Thus, Norton's argument also depends on the tacit assumption that the chances are something absolute and independent of the outcome sets, i.e., something unaffected by a relabeling of the outcomes, not just in the sense of label independence, but in the sense that the chances' own descriptions are not modified by the relabeling. I will refer to chances as absolute if they are not affected by a relabeling in this sense.

In a comparative treatment of chance, where there is no absolute chance function but only a relation of "is at most as likely as"2 $(\preccurlyeq)$, Norton's negative results fail. In fact, we will define such a relation that satisfies transitivity, reflexiveness,

Monotonicity: $\mathrm{A} \preccurlyeq \mathrm{A} \cup \mathrm{B}$,

and

Complementarity: If $\mathrm{A} \preccurlyeq \mathrm{B}$, then $\mathrm{B}^{\mathrm{C}} \preccurlyeq \mathrm{A}^{\mathrm{C}}$,

(where $\mathrm{A}^{\mathrm{C}}$ is the complement of $\mathrm{A}$ ), as well as comparative versions of label independence, finite additivity, regularity, and containment.

However, there are some limitations upon relations that have these properties. It turns out that a reasonable inductive support relation on infinite lottery outcomes cannot satisfy the standard comparative analog of countable additivity, namely,

\footnotetext{
${ }^{2}$ I have chosen this expression carefully. I did not choose the expression 'is no more likely than', as that might be taken as a synonym of 'is not more likely than', which could be regarded as true even if the chances are not comparable. Our symbol ' $\leqslant$ ' instead expresses a relation in which the events are comparable, and the first is either exactly as likely as the second or less likely.
} 
Monotone continuity: If $\mathrm{A}_{1} \subseteq \mathrm{A}_{2} \subseteq \mathrm{A}_{3} \subseteq \ldots$ and $\mathrm{A}_{1}, \mathrm{~A}_{2}, \mathrm{~A}_{3}, \ldots \preccurlyeq \mathrm{B}$ then $\mathrm{U}_{i} \mathrm{~A}_{i} \preccurlyeq \mathrm{B}$.

Nor can such a relation be total; it cannot satisfy

Comparability: For all $\mathrm{A}, \mathrm{B}$, either $\mathrm{A} \preccurlyeq \mathrm{B}$ or $\mathrm{B} \preccurlyeq \mathrm{A}$,

unless it is completely trivial. If comparability, transitivity, and comparative versions of additivity and label independence hold, then any two sets of outcomes are equally likely, so the resulting logic is utterly useless. It draws no distinctions whatsoever, not even between the impossible event $\varnothing$ and the certain event $\Omega$ (the set of all possible outcomes).

Nonetheless, if we do not require total comparability, we can have a non-trivial relation with the promised properties, and, unlike in Norton's logic, different infinite, coinfinite sets (sets with infinite complements in the sample space) can differ in likelihood. Given containment, for example, the set of outcomes labelled with multiples of four is strictly more supported than the set of even outcomes. This seems quite reasonable, given that whenever the latter set wins, the former does too, but not vice versa. Thus our comparative logic draws finer distinctions between sets of lottery outcomes than Norton's. If one is offered an opportunity to buy either all of the even-numbered tickets or all of the multiples of four for the same price, or the odd-numbered tickets versus the prime-numbered tickets, our logic indicates a clear and sensible preference.

Another reason for considering a comparative infinite lottery logic comes from Norton's own Material Theory of Induction. If the warrant for any inductive inference lies entirely in the background facts of the particular application, what background facts about a fair infinite lottery warrant the assumption that the correct logic involves a chance function? Certain comparative relationships can be justified. For example, whenever the winning ticket 
is labeled with a multiple of four, it is also labeled with an even number. Hence, drawing an even numbered ticket can be no more likely than drawing a multiple of four. If there is a chance function into some ordered structure of absolute chances that reflects such relationships, this must be shown by means of a representation theorem, or otherwise justified from considerations of what constitutes a fair infinite lottery. In this sense, comparative chance relations are more fundamental than Norton's chance function. They can, at least sometimes, be motivated directly, while the assumption that an absolute chance function exists requires further justification.

Despite its advantages, though, it does not appear that our comparative infinite lottery logic will help us in the evaluation of infinite multiverse models. It tells us, for example, that within a given model of eternal inflation, a set A of worlds is more likely to contain our world than any proper subset of $\mathrm{A}$ is. But when $\mathrm{A}$ is infinite and co-infinite, it does not tell us whether $\mathrm{A}$ is more likely than $\mathrm{A}^{\mathrm{C}}$, and hence it is not clear whether finding our world to be an A-world confirms or disconfirms the model. Furthermore, without a justifiable way of identifying worlds across models, we cannot conclude that an infinite, co-infinite set A of worlds in one model is less likely than another such set B in another model. Hence, it is not clear that the properties of our world can confirm one model over another. A relevant identification of worlds across models seems unlikely, so our comparative logic does not appear to be much more useful for confirmation than Norton's.

In any case, our comparative logic shows that Norton's infinite lottery logic is not the only one possible. There is at least one other inductive logic meeting Norton's explicit constraint (label independence) that nonetheless draws finer distinctions between outcome sets, provided we do not insist that all events are comparable, or that there are independent entities called chances. 


\section{Norton's infinite lottery logic.}

According to Norton, the "essential content" of his infinite lottery logic is given by assigning a chance value to each set of lottery outcomes, so as to define a chance function "Ch" (2018, section 8). On his view, a fair infinite lottery machine chooses among a countable infinity of outcomes "without favor", and what it is to choose without favor is specified by the requirement of label independence given above (manuscript). Previous treatments of "fair" infinite lotteries, from de Finetti (1964) to Benci et al. $(2013,2018)$, have not assumed label independence and have not arrived at Norton's logic. Instead they have supposed that fairness consists in the weaker requirement that each individual outcome has the same chance, i.e., for all $x, y \in \Omega, \operatorname{Ch}(\{x\})=\operatorname{Ch}(\{y\})$. If we assume that $\mathrm{Ch}$ is realvalued, it follows immediately from the Kolmogorov axioms of finite additivity and unit measure (i.e., $\operatorname{Ch}(\Omega)=1$ ) that $\operatorname{Ch}(\{x\})=0$ for each $x$. However, this contradicts the axiom of countable additivity, since $0+0+0+\ldots=0$, while the chance of the union $\bigcup_{x \in \Omega}\{x\}$ is $\operatorname{Ch}(\Omega)=1$. Hence de Finetti and others have rejected countable additivity for infinite lotteries, and some (e.g., Wenmackers and Horsten 2010; Benci et al. 2013; 2018) have gone further to permit hyperreal chances. The latter move allows one to avoid the conclusion that $\mathrm{Ch}(\{x\})=0$ and instead assign an infinitely small non-zero chance to each outcome, preserving regularity and containment and permitting a modified analog of countable additivity (Benci et al. 2013; 2018).

Norton (manuscript) argues that all such attempts to apply probability-like theories to fair infinite lotteries amount to changing the problem. This is because they all violate label independence, and "label independence is a defining characteristic of an infinite lottery machine" (manuscript). Historically, it seems more accurate to say that Norton has changed the problem, for only he has identified label independence as a requirement of fairness. 
Previous approaches to infinite lotteries have only required the weaker fairness condition on individual outcomes. Nonetheless, label independence holds in a fair finite lottery, and it seems sensible to extend it to the infinite case. In a finite or infinite lottery, one could plausibly buy or bet on arbitrarily many tickets in one fell swoop. Fairness then seems to require that the chance of winning such a bet does not depend on the arbitrary way in which the tickets have been numbered, and hence label independence seems to be a reasonable fairness requirement. In any case, the problem of finding the correct logic satisfying label independence seems worth considering.

As noted, this problem might be more than an interesting game. Norton argues that his infinite lottery logic is the correct logic for confirming or disconfirming eternal inflation models based on local evidence. This, however, might be doubted. Norton himself briefly considers the analogy of an array of coin flips, in which the dynamics of the coin tosses, rather than the choosing without favor of a particular coin, determine the chance of observing heads or tails. Similarly we might expect that it is the dynamics of eternal inflation and the processes by which pocket worlds emerge that determine the chance that an arbitrary world will be like ours, rather than the Principle of Mediocrity and label independence. Norton (2018) likens the coin model to "worldline based" measures discussed in the cosmology literature and sets it aside, pointing out that, according to cosmologists, such measures "exhibit an objectionable sensitivity to initial conditions" (ibid.). I think that the coin model, and the idea that the relevant chances are driven by physical dynamics rather than label independence, warrants further investigation. But the Principle of Mediocrity is taken seriously, and the debate around it is troubled by the implication that any additive measure over worlds depends on how the worlds are labeled or counted. The problem of finding a label-independent distribution over a countable infinity of worlds is entirely analogous to that 
of Norton's fair infinite lottery and is addressed by the same label-independent logic, even if that is not, in the end, the correct inductive logic for self-location in a multiverse.

In any case, Norton deduces from label-independence that any two sets of worlds, or lottery outcomes, with the same cardinality and co-cardinality must have the same chance. This is because, if two sets A and B have the same cardinality and co-cardinality, then there is a relabeling of $\Omega$ that swaps all of the labels from A to B. As discussed, the inference from this to the conclusion that $\mathrm{A}$ and $\mathrm{B}$ have the same chance depends on the assumption that each set $\mathrm{A}$ of worlds or outcomes has a chance $\mathrm{Ch}(\mathrm{A})$ whose identity is not itself affected by a relabeling. But assuming this, Norton's deduction is correct. In particular, any two subsets of $\Omega$ with the same finite cardinality $n$ must have the same chance, and Norton calls this chance $\mathrm{V}_{n}$. Likewise, any two sets with finite co-cardinality $n$ must have the same chance, which he calls $\mathrm{V}_{-n}$. In a countably infinite sample space, there remain only the countably infinite, countably co-infinite sets, which must all have the same chance $\mathrm{V}_{\infty}$. If we further assume, as Norton suggests, that that the chance of a set of outcomes is at least as great as that of any subset, we obtain a qualitative ranking of chances:

$$
\mathrm{V}_{0} \leq \mathrm{V}_{1} \leq \mathrm{V}_{2} \leq \mathrm{V}_{3} \leq \ldots \leq \mathrm{V}_{\infty} \leq \ldots \leq \mathrm{V}_{-3} \leq \mathrm{V}_{-2} \leq \mathrm{V}_{-1} \leq \mathrm{V}_{-0}
$$

It may seem natural to take these inclusive inequalities to be strict $(<)$, but on Norton's Material Theory of Induction, we must not assume that they are strict unless it is warranted by the facts of the case.

Interpreting these values, Norton (manuscript) offers a rule of coordination according to which, for finite $n \geq 0$, events of chance $\mathrm{V}_{n}$ are not to be expected, since they consist of only finitely many possible outcomes, "swamped" by the infinity of other outcomes. Similarly, events of chance $\mathrm{V}_{-n}$ are to be expected, while events of chance $\mathrm{V}_{\infty}$ are simply "as 
likely as not". Chance $\mathrm{V}_{-0}$ in particular indicates an event that is certain to happen, and $\mathrm{V}_{0}$ one that is certain not to. Though Norton does not say so, this interpretation requires that the innermost inequalities above are strict, so that $\mathrm{V}_{n}<\mathrm{V}_{\infty}<\mathrm{V}_{-n}$ for $n \geq 0$. To such extent that the facts warrant Norton's interpretive scheme, they also warrant these strict inequalities, but we will not press that point here.

It is not difficult to see that label independence contradicts finite additivity, given some familiar assumptions. However, Norton's proof can be strengthened. What Norton shows is that a finitely additive probability function on an infinite sample space cannot be label-independent. He considers a sample space $\Omega=\{1,2,3, \ldots\}$ and introduces four subsets,

$$
\begin{gathered}
\text { one }=\{1,5,9, \ldots\}, \\
\text { two }=\{2,6,10, \ldots\}, \\
\text { three }=\{3,7,11, \ldots\}, \\
\text { four }=\{4,8,12, \ldots\} .
\end{gathered}
$$

He argues that $\mathrm{Ch}($ one $)=\mathrm{Ch}($ two $)=\mathrm{Ch}($ three $)=\mathrm{Ch}($ four $)$, due to label independence. By the same token, the infinite and co-infinite set two $\cup$ three $\cup$ four must also have the same chance, i.e., $\mathrm{Ch}($ two $\cup$ three $\cup$ four $)=\mathrm{Ch}($ one $)=\mathrm{Ch}(t w o)=\ldots$. And unless all of these chances are zero, Norton says, this violates finite additivity, since for a finitely additive probability measure $\mathrm{P}$,

$$
\mathrm{P}(\text { two })+\mathrm{P}(\text { three })+\mathrm{P}(\text { four })=\mathrm{P}(\text { two } \cup \text { three } \cup \text { four }) .
$$

Norton does not explain further why Ch cannot satisfy (*), but presumably it is because ordinary addition of real (or hyperreal) numbers is strictly monotonic in the sense that $a+b>$ 
$a$ if $b>0$. Since $\mathrm{Ch}(t w o)=\mathrm{Ch}($ two $\cup$ three $\cup$ four $)$, Ch cannot satisfy $(*)$ unless $\mathrm{Ch}($ three $)=$ $\mathrm{Ch}($ four $)=0$. And in that case we also get $\mathrm{Ch}($ one $)=\mathrm{Ch}($ two $)=\mathrm{Ch}($ two $\cup$ three $\cup$ four $)=0$. Hence, $\left(^{*}\right)$, and therefore finite additivity, must be false unless all of these chances are zero. And, we may add, the latter possibility is both implausible and unhelpful. If all of these chances are zero, then finite additivity would imply that $\mathrm{Ch}(\Omega)=\mathrm{Ch}($ one $)+\mathrm{Ch}($ two $)+$ $\mathrm{Ch}($ three $)+\mathrm{Ch}($ four $)=0$. This contradicts the unit measure axiom of probability $(\mathrm{P}(\Omega)=1)$ and further makes the certain event $\Omega$ and the impossible event $\varnothing$ equally likely, which is absurd.

Thus Norton shows conclusively that $\mathrm{Ch}$ is not a finitely additive, real-valued or even hyperreal-valued probability. The reason I say that Norton's proof can be strengthened is because Norton's chances are not real or hyperreal numbers. Hence we might suppose that they have an addition operation that is not strictly monotonic but satisfies finite additivity. After all, Cantor's cardinal numbers have such an addition operation. The sum of two cardinals $c$ and $d$ is just the maximum of the two if either cardinal is infinite. And since the chance of a set of lottery outcomes is here determined by the cardinalities of the set and its complement, it would not be surprising if these chances had an arithmetic similar to cardinal arithmetic. So we might hope to define chance addition in such a way that strict monotonicity fails and additivity holds.

However, this cannot be done without trivializing chances. We can show that there is no well defined binary operation '+' on Norton's chances $\mathrm{V}_{i}$ that satisfies finite additivity unless all the $\mathrm{V}_{i}$ are identical (for $i=0,1,2, \ldots, \infty, \ldots,-2,-1,-0$ ). For, we can show that if both label independence and finite additivity hold, then all infinite sets have the same chance (Proposition 1(1) in the Appendix). This is untenable, given that an infinite, co-infinite set of outcomes is as likely as not, while an infinite, co-finite set is to be expected. Furthermore, if we make the minimally rational assumption that the complement of an event that is certain to 
occur is certain not to occur, then label independence and finite additivity imply that all coinfinite sets also have the same chance. Thus, by transitivity of identity, all sets of outcomes have the same chance (Proposition 1(2)). This trivializes chances and, again, is utterly implausible, for it implies that the empty set of outcomes, which cannot occur, has the same chance as the set of all possible outcomes, which must occur. Hence, finite additivity fails unavoidably for any reasonable chance function on a label-independent, countably infinite lottery.

It is easy to see that containment also fails for Norton's chance function. For example, if $\Omega=\{1,2,3, \ldots\}$ and $A=\{4,8,16, \ldots\}$, then $A$ is a proper subset of $B=\{2,4$, $6, \ldots\}$, so containment implies that A must have strictly smaller chance than B. But A and B are both infinite and co-infinite, so label independence implies that they must have the same chance.

\section{A comparative infinite lottery logic.}

Let us now see what can be gained if we sacrifice the notion of a chance function and absolute chances. Let $\Omega$ be a set of possible lottery outcomes. For any two sets $\mathrm{A}, \mathrm{B} \subseteq \Omega$, let

$$
\mathrm{A} \preccurlyeq \mathrm{B} \Leftrightarrow \mathrm{A} \backslash \mathrm{B} \text { is finite and }|\mathrm{A} \backslash \mathrm{B}| \leq|\mathrm{B} \backslash \mathrm{A}| \text {, }
$$

where ' $\mathrm{A} \backslash \mathrm{B}$ ' denotes set difference $\mathrm{A} \cap\left(\mathrm{B}^{\mathrm{C}}\right)$ and ' $|\cdot|$ ' denotes Cantorian cardinality. The condition that $\mathrm{A} \backslash \mathrm{B}$ is finite is needed here if $\Omega$ is infinite, because without that stipulation we would have $\mathrm{A} \preccurlyeq \mathrm{B}$ for any disjoint countably infinite sets $\mathrm{A}$ and $\mathrm{B}$, entailing violations of either additivity and containment or transitivity.

Norton's infinite lottery logic is derived from his analysis of the "background facts" of a fair infinite lottery, especially the requirement of label independence. Our project here is 
somewhat different; we primarily want to show that there exists at least one alternative inductive logic that is not ruled out by those background facts, and that such a logic could have many nice properties that Norton's logic lacks. Thus our relation $\preccurlyeq$ is presented first and foremost as an example of a comparative logic that does not violate label independence. Its primary philosophical interest lies in showing that, if we do not assume that outcome sets have absolute chances, then label independence does not contradict additivity. Whether the background facts of an infinite lottery, or eternal inflation, do warrant our particular comparative logic is another question.

However, some motivation for our particular comparative logic can be given. The 'if' direction of our definition of ' $\preccurlyeq$ ' does seem to be warranted, for, if one difference $A \backslash B$ or B $\backslash \mathrm{A}$ is strictly smaller than the other, then it is plausible that the former difference is at most as likely as the latter. Furthermore, since A and B are, tautologically, the same except for their differences, the relative likelihoods of those differences should determine the relative likelihoods of $\mathrm{A}$ and $\mathrm{B} .^{3}$ The condition that one of the differences is finite just guarantees that the relative sizes and likelihoods of the differences are clear. Even on Norton's system, a finite outcome set has smaller chance than an infinite one. It therefore seems reasonable that the set with a finite difference should also have a smaller chance than the set with an infinite difference or a larger finite difference.

Whether the 'only if' direction is warranted is essentially the question of whether two sets with infinite differences (which are therefore countably infinite and co-infinite) should be regarded as incomparable or equal in support. We may ask whether the assumption of incomparability is warranted, but it would seem that the greater burden lies on one who holds that comparability is warranted. Our relation declines to comment on pairs of sets with infinite differences, whereas a total relation that makes them equal would require

\footnotetext{
${ }^{3}$ This intuition is equivalent to the comparative version of additivity, discussed below.
} 
some warrant for doing so. Again, label independence may seem to suggest that sets related by a relabeling are equally likely, but if we do not assume that events have absolute chances, then label independence is compatible with some such sets being merely incomparable. And again, it is not clear that the assumption of absolute chances is warranted by the facts of a fair infinite lottery. Hence, our chance relation seems to be better warranted than Norton's chance function, as it involves fewer assumptions. The philosophical motivation for requiring that the smaller difference be finite in our definition of ' $\preccurlyeq$ ' is simply that, in this context, if both differences are infinite, they have the same cardinality and co-cardinality, and hence we have no clear basis for comparison.

Let us now see what properties our partial relation ' $\preccurlyeq$ ' has. First, note that it is transitive, reflexive, and monotonic (Propositions 2-4 in the Appendix).

Now let us formulate a precise version of label independence for relations. A labeling of $\Omega$ is a bijection $l: \mathbf{N} \rightarrow \Omega$. Given $\mathrm{X} \subseteq \mathbf{N}$, let us write $l \mathrm{X}$ for the set $\{l(x): x \in \mathrm{X}\} \subseteq \Omega$. We can now define

Label independence': For any $\mathrm{X}, \mathrm{Y} \subseteq \mathbf{N}$ and labelings $l_{1}, l_{2}$,

$$
l_{1} \mathrm{X} \preccurlyeq l_{1} \mathrm{Y} \Leftrightarrow l_{2} \mathrm{X} \preccurlyeq l_{2} \mathrm{Y}
$$

This is logically weaker than label independence proper, but it is clearly equivalent to label independence in a context where anything we can say about the chances is equivalent to some collection of statements that are each of the form $X \preccurlyeq Y$ or $\neg(X \preccurlyeq Y)$. And, indeed, our relation ' $\preccurlyeq$ ' is label-independent' (Proposition 5).

A standard comparative analog of finite additivity going back to de Finetti (1964, originally 1937) is 
Additivity': If $\mathrm{A} \cap \mathrm{C}=\mathrm{B} \cap \mathrm{C}=\varnothing$, then $\mathrm{A} \preccurlyeq \mathrm{B} \Leftrightarrow \mathrm{A} \cup \mathrm{C} \preccurlyeq \mathrm{B} \cup \mathrm{C}$.

Though this property is commonly called additivity in the literature (e.g., Fishburn 1986), its precise relationship to finite additivity for absolute probabilities is not obvious. Kraft et al. (1959) showed that it does not imply the existence of a finitely additive probability measure on $\Omega$ that "almost agrees" with ' $\preccurlyeq$ ' in the sense that $\mathrm{A} \preccurlyeq \mathrm{B} \Rightarrow \mathrm{P}(\mathrm{A}) \leq \mathrm{P}(\mathrm{B})$. However, it does imply that we can construct abstract chances with an operation that satisfies finite additivity. Let us write ' $\mathrm{A} \sim \mathrm{B}$ ' for ' $\mathrm{A} \preccurlyeq \mathrm{B}$ and $\mathrm{B} \preccurlyeq \mathrm{A}$ '. Let $\mathrm{Ch}(\mathrm{A})=[\mathrm{A}]=\{\mathrm{X} \subseteq \Omega: \mathrm{X} \sim \mathrm{A}\}$, and define addition by $[\mathrm{A}]+[\mathrm{B}]=[\mathrm{A} \cup \mathrm{B}]$ where $\mathrm{A} \cap \mathrm{B}=\varnothing$. Additivity' guarantees that this is well defined, for if $[C]=[A]$ and $[D]=[B]$ then $[C \cup D]=[A \cup B]$ (Proposition 6). This way of defining Ch, taking equivalence classes of outcome sets as values, does not undermine finite additivity, because it does not license us to infer that, given a permutation $p$ of $\Omega, \operatorname{Ch}(\mathrm{A})=\mathrm{Ch}(p \mathrm{~A})$. It instead licenses us to infer from $\mathrm{Ch}(\mathrm{A})=[\mathrm{A}]$ that $\mathrm{Ch}(p \mathrm{~A})=[p \mathrm{~A}]$, but that is trivial and consistent with additivity.

Thus, additivity' does imply that finite additivity is satisfiable. And, as it happens, our 'ミ’ is additive' (Proposition 7).

It is obvious how to formulate the comparative version of containment:

Containment': $\mathrm{A} \subset \mathrm{B} \Rightarrow \mathrm{A} \prec \mathrm{B}$,

where ' $\mathrm{A} \prec \mathrm{B}$ ' is shorthand for ' $\mathrm{A} \preccurlyeq \mathrm{B}$ and it is not the case that $\mathrm{B} \preccurlyeq \mathrm{A}$ '. Norton (manuscript) suggests that the chance of a set of outcomes cannot be diminished if we add further outcomes to the set, so that the relation 'is at most as strong as' on chances inherits the properties of set theoretic inclusion. Prima facie, it seems just as plausible that the chance of a set of outcomes is actually increased if we add further outcomes to the set. In fact, those 
who hold that chances should satisfy both finite additivity and regularity must accept containment' as a consequence. Since permutations preserve set inclusion, one might expect that containment' is compatible with label independence', so it is not too surprising that our chance relation does satisfy containment' (Proposition 8).

In Norton's logic, all infinite, co-infinite sets have the same chance. Containment' implies that our chance relation draws finer distinctions between sets of outcomes. For example, if we again label the outcomes $1,2,3, \ldots$, then $\{4,8,12, ..\} \prec\{2,4,6, \ldots\}$. As well, prime $=\{n: n$ is prime $\} \prec$ odd $=\{n: n$ is odd $\}$, since prime $\backslash$ odd $=\{2\}$ while odd $\backslash$ prime is infinite.

Though it is controversial, many have argued that chances should be regular, for various reasons. (See Benci et al. 2018 for discussion and references.) A natural comparative analog of regularity is

$$
\text { Regularity': If } \mathrm{B} \neq \varnothing \text { then } \varnothing \prec \mathrm{B} \text {. }
$$

In Norton's ordering of chances, we have $\mathrm{Ch}(\varnothing) \leq \mathrm{Ch}(\mathrm{B})$, but Norton is studiously silent on whether $\mathrm{Ch}(\mathrm{B}) \leq \mathrm{Ch}(\varnothing)$ for $\mathrm{B} \neq \varnothing$. If this is never the case, then the ordering of sets induced by their chances is regular', and if not, not. However, our relation ' $\preccurlyeq$ ' is explicitly regular'. (Proposition 9). In fact, regularity' is just the special case of containment' where $A=\varnothing$.

Complementarity is an eminently reasonable property for a chance relation to have, for an event is likely just insofar as its non-occurrence is unlikely. So it stands to reason that an event $\mathrm{A}$ is at most as likely as $\mathrm{B}$ if and only $\mathrm{B}^{\mathrm{C}}$ is at most as likely as $\mathrm{A}^{\mathrm{C}}$. Happily, our relation has this property too (Proposition 10).

Thus, our relation ' $\preccurlyeq$ ' has all the nice properties promised. 


\section{Limitations of comparative infinite lottery logic.}

Villegas (1964) identified monotone continuity as the key property required for there to be a countably additive probability function agreeing with a given relation. (Our slightly generalized version of monotone continuity above follows Fishburn (1986)). However, any reasonable chance relation for a fair infinite lottery, including our ' $\preccurlyeq$ ', must lack this property. In particular, we assume that a chance relation in this context should satisfy complementarity and

Infinity dominance: If $\mathrm{A}$ is finite and $\mathrm{B}$ infinite, then $\mathrm{A} \preccurlyeq \mathrm{B}$ and $\varnothing \prec \mathrm{B}$.

Proposition 11 tells us that such a relation on the subsets of a countably infinite outcome space cannot be monotonically continuous. ${ }^{4}$

By definition, our chance relation ' $\preccurlyeq$ ' does not satisfy comparability, but we might wonder whether some chance relation could satisfy comparability without sacrificing the other nice properties we have established. The answer is no. Much as finite additivity would imply that Norton's chances are all equal, we can show that if a chance relation satisfies transitivity, label independence', additivity', and comparability, it is just the trivial relation that holds between any two sets (Proposition 12).

\section{Application to multiverse cosmologies.}

There are various models of inflation (e.g., "slow roll” versus "bubble nucleation”), and the way in which properties are distributed over worlds is model-dependent (Vilenkin

\footnotetext{
${ }^{4}$ DiBella (2018) shows that, under Koopman's (1940) axioms of comparative conditional confidence, monotone continuity is inconsistent with the weak fairness condition that $\{x\} \sim\{y\}$ for each $x, y \in \Omega$.
} 
1995). The problem with applying Norton's logic to eternal inflation was that, for any model according to which there are infinitely many worlds like ours and infinitely many unlike it, the chance of finding ourselves in a world like ours is, on his logic, simply "as likely as not". Any infinite, co-infinite set of worlds is as likely as any other to contain the world we observe, setting aside selection effects. So, even if, on some measure, our world is extremely typical or atypical in a given model, observing a world like ours does not make the model any more or less likely to be correct. Furthermore, such an observation does not confirm one model of inflation any more than it confirms another. (Of course, there may be other kinds of locally available evidence, depending on the details of the theory. See Vilenkin 2011 for some discussion.)

Our comparative logic does not have quite the same problem. On our logic, some properties of worlds are more likely than others, even though both properties hold in infinitely many worlds and fail in infinitely many worlds. For example, suppose some property $\mathrm{P}$ holds in a countable infinity of worlds and fails in a countable infinity of worlds, while property Q holds in a countably infinite proper subset of those having P. For Norton, we are exactly as likely to observe P as Q (again, setting aside any selection effects). Under our comparative logic, we are more likely to observe P than Q, in virtue of containment'.

Unfortunately, this appears to be little help if we want to evaluate the inductive support for a given model of eternal inflation. We can deduce that one observation is more confirming than another, but this does not tell us whether to regard either observation as incrementally confirming or disconfirming the model. Nor does our logic seem to help with evaluating multiple models relative to each other. Suppose we observe property $\mathrm{P}$ in our world. What we need in order to infer that a model $\mathrm{M}$ is better confirmed than a model $\mathrm{N}$ is to show that $\mathrm{M}$ makes $\mathrm{P}$ more likely than $\mathrm{N}$ does. But our comparative chance relation does not extend across models. The models $\mathrm{M}$ and $\mathrm{N}$ will simply contain different worlds, and our 
relation can say nothing about how an infinite, co-infinite set of worlds in one model compares, in terms of inductive support, to one in another model. Nor can we identify a set of worlds in one model with a set of worlds in another. In general, the models will not even contain specific worlds, but only tell us something about how properties are distributed among the worlds generated by some stochastic process.

If two models do contain specific worlds, we might consider identifying the most similar counterparts across models, much as Lewis does in his possible worlds semantics for modality (1968), but this does not appear to work. Outside the semantic demands of modal locutions, there is no clear reason to identify counterparts across models, nor to attribute to such counterpart relations any evidentiary value. And anyway, such a move would tend to identify worlds that have the same properties, so that, from one model to another, there would be little difference between the ways properties are distributed over the worlds so identified.

On the other hand, it might be the case that a model M makes a property P more likely than another property $\mathrm{Q}$, while model $\mathrm{N}$ makes them equally likely, or at least makes 'P and not Q' very unlikely because $\{\mathrm{P}$-worlds $\} \backslash\{\mathrm{Q}$-worlds $\}$ is finite. Then if we observe $\mathrm{P}$ and not Q, this would seem to be some evidence for M over N. But we are extremely unlikely to observe P-and-not-Q unless, in the real universe, $\{\mathrm{P}$-worlds $\} \backslash\{\mathrm{Q}$-worlds $\}$ is infinite. Hence the evidence P-and-not-Q would strongly disconfirm both models unless, in model $\mathrm{M},\{\mathrm{P}-$ worlds $\} \backslash\{\mathrm{Q}$-worlds $\}$ is infinite. But in that case we have observed a property, P-and-not-Q, that is instantiated by infinitely many worlds in model $\mathrm{M}$ and only finitely many in model $\mathrm{N}$. Then indeed, model $\mathrm{M}$ is better confirmed, but that is a case that even Norton's logic can handle. What we want is a logic that can help us when the evidence consists in properties that are instantiated in an infinite, co-infinite set of worlds in every model under consideration. And that sort of logic, it appears, we still do not have. 
One lesson we can take away from this is that the difficulty of applying label independence to self-location in an infinite multiverse runs deeper than the particular formal oddities of Norton's absolute logic, namely the failure of finite additivity and the equality of all infinite, co-infinite sets. Even a comparative inductive support relation satisfying label independence, additivity, and containment does not help us to evaluate multiverse theories based on self-locating belief, for it provides no basis for comparing infinite sets that have infinite differences. While label independence' on its own does not guarantee that infinite, co-infinite sets have equal inductive support, as in Norton's logic, it does block us from judging one such set to be strictly better supported than another when the sets are disjoint. This makes comparisons between a set of worlds and its complement, or between sets of worlds within different models, unhelpful for evaluating multiverse theories.

\section{Conclusion.}

Norton's infinite lottery logic is radically different from and weaker than standard inductive logic or probability theory. The chief difference that Norton emphasizes is the failure of finite additivity. However, in a comparative infinite lottery logic, satisfying a comparative analog of Norton's key principle of label independence, we can have a comparative version of additivity, and we can even construct chances (equivalence classes of events) that have an additive operation without violating label independence. We can also have transitivity, reflexiveness, monotonocity, and comparative analogs of regularity and containment. These properties enable us to draw finer distinctions of likelihood between sets of outcomes for fair, label-independent infinite lotteries. And yet, we may still lack a useful tool for evaluating multiverse theories on the basis of local features of the world. Without a way of identifying worlds across different models, our logic does not seem to provide a means of determining which multiverse theories make worlds like ours more likely. What it 
does provide is an illustration of the fact that Norton's infinite lottery logic is not the only one possible under his strong fairness condition. If we sacrifice total comparability and the notion of independent entities called chances, we can recover additivity, as well as many other nice properties, and draw finer distinctions between outcome sets than Norton's logic does. 


\section{Appendix: Proofs.}

Proposition 1: Given a label-independent and finitely additive function Ch on a countably infinite sample space $\Omega$,

(1) $\mathrm{Ch}(\mathrm{A})=\mathrm{Ch}(\mathrm{B})$ for all infinite sets $\mathrm{A}, \mathrm{B} \subseteq \Omega$, and

(2) if for all $\mathrm{A} \subseteq \Omega$, either $\operatorname{Ch}(\mathrm{A}) \neq \mathrm{Ch}(\Omega)$ or $\operatorname{Ch}\left(\mathrm{A}^{\mathrm{C}}\right)=\mathrm{Ch}(\varnothing)$, then $\mathrm{Ch}(\mathrm{B})=$ $\mathrm{Ch}(\mathrm{C})$ for any $\mathrm{B}, \mathrm{C} \subseteq \Omega$.

Proof: (1) Suppose $A, B \subseteq \Omega$ are infinite. Partition each into two infinite, co-infinite sets $C$, $\mathrm{D} \subseteq \mathrm{A}$ and $\mathrm{E}, \mathrm{F} \subseteq \mathrm{B}$, so that $\mathrm{C} \cap \mathrm{D}=\mathrm{E} \cap \mathrm{F}=\varnothing, \mathrm{C} \cup \mathrm{D}=\mathrm{A}$, and $\mathrm{E} \cup \mathrm{F}=\mathrm{B}$. Since $\mathrm{C}, \mathrm{D}, \mathrm{E}$, $\mathrm{F}$ are all infinite and co-infinite, they all have the same chance, by label independence. By finite additivity, $\mathrm{Ch}(\mathrm{A})=\mathrm{Ch}(\mathrm{C})+\mathrm{Ch}(\mathrm{D})=\mathrm{Ch}(\mathrm{E})+\mathrm{Ch}(\mathrm{F})=\mathrm{Ch}(\mathrm{B})$.

(2) Assume that for all $\mathrm{A} \subseteq \Omega, \operatorname{Ch}(\mathrm{A}) \neq \mathrm{Ch}(\Omega)$ or $\operatorname{Ch}\left(\mathrm{A}^{\mathrm{C}}\right)=\mathrm{Ch}(\varnothing)$. We first show that for any co-infinite $\mathrm{D}, \mathrm{E} \subseteq \Omega, \mathrm{Ch}(\mathrm{D})=\mathrm{Ch}(\mathrm{E})$. Let $\mathrm{D}, \mathrm{E} \subseteq \Omega$ be co-infinite. Then by (1), $\mathrm{Ch}\left(\mathrm{D}^{\mathrm{C}}\right)=\mathrm{Ch}\left(\mathrm{E}^{\mathrm{C}}\right)=\mathrm{Ch}(\Omega)$. By our assumption, then, $\mathrm{Ch}(\mathrm{D})=\mathrm{Ch}(\mathrm{E})=\mathrm{Ch}(\varnothing)$. So all coinfinite sets have equal chance, and as we already saw, all infinite sets have equal chance. But all subsets of $\Omega$ are either infinite or co-infinite, and some are both, so by transitivity of identity, $\mathrm{Ch}(\mathrm{B})=\mathrm{Ch}(\mathrm{C})$ for any $\mathrm{B}, \mathrm{C} \subseteq \Omega$.

Proposition 2: ' $\preccurlyeq$ ' is transitive.

Proof: Assume $\mathrm{A} \preccurlyeq \mathrm{B}$ and $\mathrm{B} \preccurlyeq \mathrm{C}$. Then by definition of $\preccurlyeq, \mathrm{A} \backslash \mathrm{B}$ and $\mathrm{B} \backslash \mathrm{C}$ are finite, $|\mathrm{A} \backslash \mathrm{B}| \leq|\mathrm{B} \backslash \mathrm{A}|$, and $|\mathrm{B} \backslash \mathrm{C}| \leq|\mathrm{C} \backslash \mathrm{B}|$, where '|.|' denotes Cantorian cardinality. We need to show that $\mathrm{A} \preccurlyeq \mathrm{C}$, i.e., $\mathrm{A} \backslash \mathrm{C}$ is finite and $|\mathrm{A} \backslash \mathrm{C}| \leq|\mathrm{C} \backslash \mathrm{A}|$. Let us partition $\mathrm{A}, \mathrm{B}$, and $\mathrm{C}$ into necessarily disjoint subsets. (For brevity we write ' $\mathrm{XY}$ ' for ' $\mathrm{X} \cup \mathrm{Y}$ '.) Let

$$
\mathrm{a}=\mathrm{A} \backslash(\mathrm{BC}), \quad \mathrm{b}=\mathrm{B} \backslash(\mathrm{AC}),
$$




$$
\begin{array}{ll}
\mathrm{c}=\mathrm{C} \backslash(\mathrm{AB}), & \mathrm{e}=\mathrm{B} \cap \mathrm{C} \backslash \mathrm{A}, \\
\mathrm{d}=\mathrm{A} \cap \mathrm{B} \backslash \mathrm{C}, & \mathrm{f}=\mathrm{A} \cap \mathrm{C} \backslash \mathrm{B} .
\end{array}
$$

(See Figure 1.) Then a, b, c, d, e, f are all pairwise disjoint.

Note $\mathrm{A} \backslash \mathrm{B}=\mathrm{af}, \mathrm{B} \backslash \mathrm{A}=\mathrm{be}, \mathrm{B} \backslash \mathrm{C}=\mathrm{bd}, \mathrm{C} \backslash \mathrm{B}=\mathrm{cf}, \mathrm{A} \backslash \mathrm{C}=\mathrm{ad}$, and $\mathrm{C} \backslash \mathrm{A}=$ ce.

$\mathrm{A} \backslash \mathrm{C}=\mathrm{ad}$ is finite because $\mathrm{A} \backslash \mathrm{B}=\mathrm{af}$ and $\mathrm{B} \backslash \mathrm{C}=\mathrm{bd}$ are finite. It only remains to show that $|\mathrm{ad}| \leq|\mathrm{ce}|$

Adding our two inequalities, $|\mathrm{A} \backslash \mathrm{B}| \leq|\mathrm{B} \backslash \mathrm{A}|$, and $|\mathrm{B} \backslash \mathrm{C}| \leq|\mathrm{C} \backslash \mathrm{B}|$, we get

$$
|a f|+|b d| \leq|b e|+|c f| .
$$

Hence $|\operatorname{adbf}| \leq \mid$ cebf $\mid$, so

$$
|\mathrm{ad}|+|\mathrm{bf}| \leq|\mathrm{ce}|+|\mathrm{bf}| .
$$

In general, subtraction is not defined for cardinals, but it is for finite cardinals. We know that $\mathrm{A} \backslash \mathrm{B}$ and $\mathrm{B} \backslash \mathrm{C}$ are finite. Therefore, $\mathrm{a}, \mathrm{d}, \mathrm{f}$, and $\mathrm{b}$ are finite.

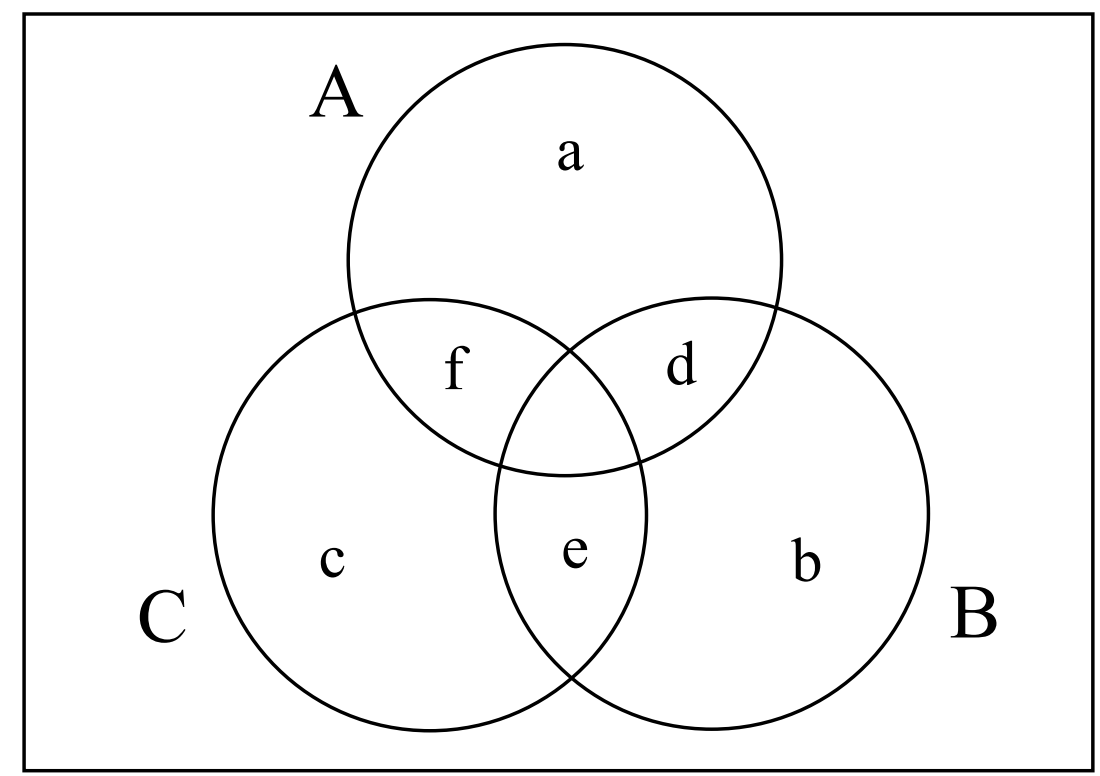

Figure 1. The sets defined in the proof of Proposition 2. 
If $\mathrm{c}$ or e is infinite, then $|\mathrm{ad}| \leq|\mathrm{ce}|$ and the claim holds.

If $\mathrm{c}$ and e are both finite, then subtraction applies. Subtracting $|\mathrm{bf}|$ from the inequality $(* *),|\operatorname{ad}| \leq \mid$ ce $\mid$. Therefore, $\mathrm{A} \backslash \mathrm{C}=\operatorname{ad}$ is finite and $|\mathrm{A} \backslash \mathrm{C}| \leq|\mathrm{C} \backslash \mathrm{A}|$, so $\mathrm{A} \preccurlyeq \mathrm{C}$.

Proposition 3: ' $\preccurlyeq$ ' is reflexive.

Proof: $A \preccurlyeq A \Leftrightarrow(A \backslash A$ is finite and $|A \backslash A| \leq|A \backslash A|)$. Since $A \backslash A=\varnothing$ and $0 \leq 0$, this is true.

Proposition 4: ' $\preccurlyeq$ ' is monotonic.

Proof: $\mathrm{A} \preccurlyeq \mathrm{A} \cup \mathrm{B} \Leftrightarrow(\mathrm{A} \backslash(\mathrm{A} \cup \mathrm{B})$ is finite and $|\mathrm{A} \backslash(\mathrm{A} \cup \mathrm{B})| \leq|(\mathrm{A} \cup \mathrm{B}) \backslash \mathrm{A}|)$. But $|\mathrm{A} \backslash(\mathrm{A} \cup \mathrm{B})|=0 \leq|(\mathrm{A} \cup \mathrm{B}) \backslash \mathrm{A}|$, so the claim holds.

Lemma 1: A relation $\preccurlyeq$ on the subsets of a countably infinite set $\Omega$ is label-independent' if and only if for any permutation $p: \Omega \rightarrow \Omega$, and any $\mathrm{A}, \mathrm{B} \subseteq \Omega$,

$$
\mathrm{A} \preccurlyeq \mathrm{B} \Leftrightarrow p \mathrm{~A} \preccurlyeq p \mathrm{~B}
$$

Proof: If $\preccurlyeq$ is label-independent', then for any labelings $l_{1}$ and $l_{2}$,

$$
l_{1} \mathrm{~A} \preccurlyeq l_{1} \mathrm{~B} \Leftrightarrow l_{2} \mathrm{~A} \preccurlyeq l_{2} \mathrm{~B} .
$$

For any permutation $p: \Omega \rightarrow \Omega$, there exist labelings $l_{1}, l_{2}$ such that $p=l_{2} \circ l_{1}{ }^{-1}$.

$$
\begin{gathered}
\text { So } \mathrm{A} \preccurlyeq \mathrm{B} \Leftrightarrow l_{1}\left(l_{1}^{-1} \mathrm{~A}\right) \preccurlyeq l_{1}\left(l_{1}{ }^{-1} \mathrm{~B}\right) \\
\Leftrightarrow l_{2}\left(l_{1}^{-1} \mathrm{~A}\right) \preccurlyeq l_{2}\left(l_{1}^{-1} \mathrm{~B}\right) \\
\Leftrightarrow p \mathrm{~A} \preccurlyeq p \mathrm{~B} .
\end{gathered}
$$


Conversely, if $\mathrm{A} \preccurlyeq \mathrm{B} \Leftrightarrow p \mathrm{~A} \preccurlyeq p \mathrm{~B}$, and $l_{1}, l_{2}$ are labelings, let $p=l_{2} \circ l_{1}{ }^{-1}$.

Then $l_{1} \mathrm{X} \preccurlyeq l_{1} \mathrm{Y} \Leftrightarrow p\left(l_{1} \mathrm{X}\right) \preccurlyeq p\left(l_{1} \mathrm{Y}\right)$

$$
\begin{aligned}
& \Leftrightarrow l_{2}\left(l _ { 1 } ^ { - 1 } ( l _ { 1 } \mathrm { X } ) \preccurlyeq l _ { 2 } \left(l_{1}^{-1}\left(l_{1} \mathrm{Y}\right)\right.\right. \\
& \Leftrightarrow l_{2} \mathrm{X} \preccurlyeq l_{2} \mathrm{Y} .
\end{aligned}
$$

Proposition 5: ' $\preccurlyeq$ ' is label-independent'.

Proof: Let $p$ be a permutation of $\Omega$. Since permutations preserve cardinality,

$$
\begin{aligned}
\mathrm{A} \preccurlyeq \mathrm{B} & \Leftrightarrow \mathrm{A} \backslash \mathrm{B} \text { is finite and }|\mathrm{A} \backslash \mathrm{B}| \leq|\mathrm{B} \backslash \mathrm{A}| \\
& \Leftrightarrow p(\mathrm{~A} \backslash \mathrm{B}) \text { is finite and }|p(\mathrm{~A} \backslash \mathrm{B})| \leq|p(\mathrm{~B} \backslash \mathrm{A})| \\
& \Leftrightarrow p \mathrm{~A} \backslash p \mathrm{~B} \text { is finite and }|p \mathrm{~A} \backslash p \mathrm{~B}| \leq|p \mathrm{~B} \backslash p \mathrm{~A}| \\
& \Leftrightarrow p \mathrm{~A} \preccurlyeq p \mathrm{~B} .
\end{aligned}
$$

By Lemma 1, it follows that ' $\preccurlyeq$ ' is label-independent'.

Proposition 6: For any transitive, reflexive, and additive' relation ' $\preccurlyeq$ ' on sets (and the induced relation ' $\sim$ '), if $\mathrm{A} \cap \mathrm{B}=\mathrm{C} \cap \mathrm{D}=\varnothing, \mathrm{A} \sim \mathrm{C}$, and $\mathrm{B} \sim \mathrm{D}$, then $\mathrm{A} \cup \mathrm{B} \sim \mathrm{C} \cup \mathrm{D}$.

Proof: Assume that $\mathrm{A} \cap \mathrm{B}=\mathrm{C} \cap \mathrm{D}=\varnothing, \mathrm{A} \sim \mathrm{C}$, and $\mathrm{B} \sim \mathrm{D}$. Let us partition $\mathrm{A}, \mathrm{B}, \mathrm{C}$, and $\mathrm{D}$ into disjoint components. Again we write $\mathrm{XY}$ for $\mathrm{X} \cup \mathrm{Y}$. Let

$$
\begin{array}{ll}
\mathrm{a}=\mathrm{A} \backslash(\mathrm{CD}), & \mathrm{e}=\mathrm{A} \cap \mathrm{C}, \\
\mathrm{b}=\mathrm{B} \backslash(\mathrm{CD}), & \mathrm{f}=\mathrm{B} \cap \mathrm{C}, \\
\mathrm{c}=\mathrm{C} \backslash(\mathrm{AB}), & \mathrm{g}=\mathrm{B} \cap \mathrm{D}, \\
\mathrm{d}=\mathrm{D} \backslash(\mathrm{AB}), & \mathrm{h}=\mathrm{A} \cap \mathrm{D} .
\end{array}
$$


(See Figure 2.) We want to show that abefgh $\sim$ cdefgh. By additivity', it is enough to show that $\mathrm{ab} \sim \mathrm{cd}$. Since B $\sim \mathrm{D}, \mathrm{bfg} \sim \mathrm{dgh}$. By additivity', bf $\sim$ dh and hence

$$
\text { abf } \sim \text { adh. }
$$

Since A $\sim \mathrm{C}$, aeh $\sim$ cef, additivity' implies that ah $\sim \mathrm{cf}$ and hence

$$
\text { adh } \sim \text { cdf. }
$$

By transitivity, abf $\sim$ cdf, so by additivity' again, $\mathrm{ab} \sim \mathrm{cd}$. Therefore, $\mathrm{A} \cup \mathrm{B} \sim \mathrm{C} \cup \mathrm{D}$.

Proposition 7: ' $\preccurlyeq$ ' is additive'.

Proof: Assume $\mathrm{A} \cap \mathrm{C}=\mathrm{B} \cap \mathrm{C}=\varnothing$. Then $\mathrm{A} \backslash \mathrm{B}=(\mathrm{A} \cup \mathrm{C}) \backslash(\mathrm{B} \cup \mathrm{C})$ and $\mathrm{B} \backslash \mathrm{A}=(\mathrm{B} \cup \mathrm{C}) \backslash$ $(A \cup C)$. Therefore,

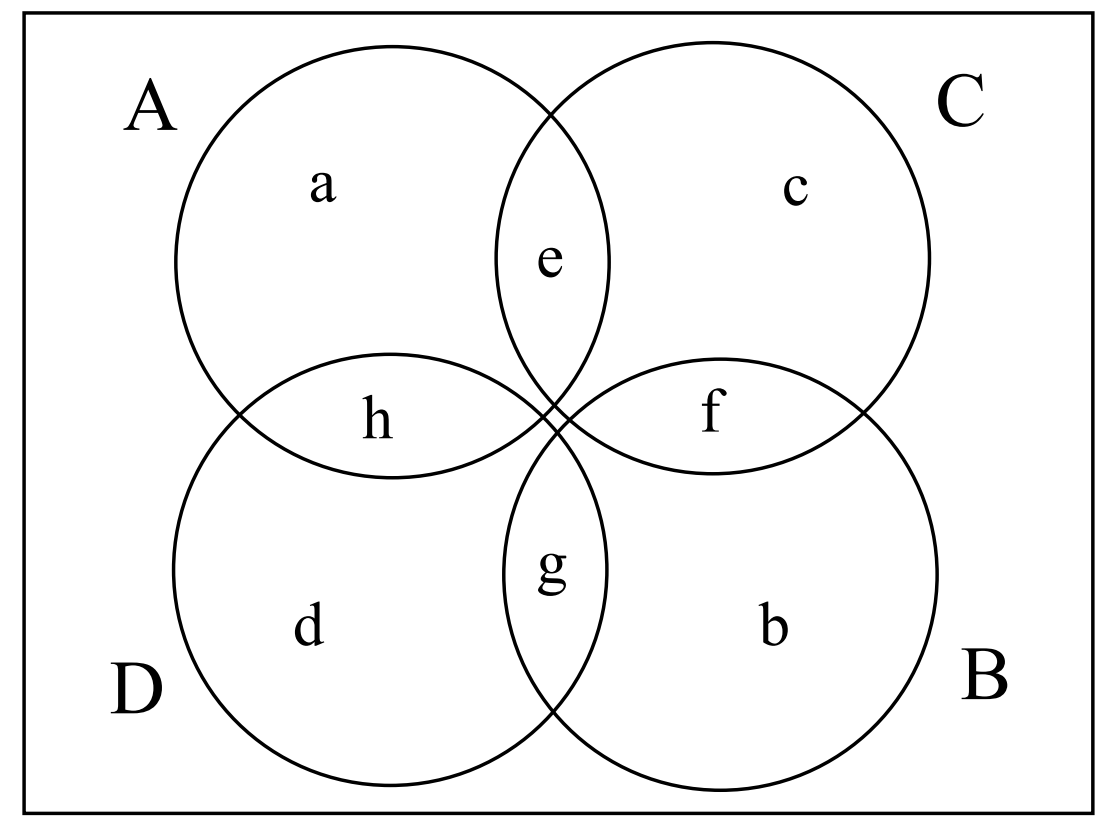

Figure 2. The sets defined in the proof of Proposition 6. 


$$
\begin{aligned}
\mathrm{A} \preccurlyeq \mathrm{B} & \Leftrightarrow(\mathrm{A} \cup \mathrm{C}) \backslash(\mathrm{B} \cup \mathrm{C}) \text { is finite and }|(\mathrm{A} \cup \mathrm{C}) \backslash(\mathrm{B} \cup \mathrm{C})| \leq|(\mathrm{B} \cup \mathrm{C}) \backslash(\mathrm{A} \cup \mathrm{C})| \\
& \Leftrightarrow \mathrm{A} \cup \mathrm{C} \preccurlyeq \mathrm{B} \cup \mathrm{C} .
\end{aligned}
$$

Proposition 8: ' $\preccurlyeq$ ' satisfies containment'.

Proof: $\mathrm{A} \subset \mathrm{B} \Rightarrow|\mathrm{A} \backslash \mathrm{B}|=0$ and $|\mathrm{B} \backslash \mathrm{A}|>0$

$\Rightarrow \mathrm{A} \backslash \mathrm{B}$ is finite, $|\mathrm{A} \backslash \mathrm{B}| \leq|\mathrm{B} \backslash \mathrm{A}|$, and not $|\mathrm{B} \backslash \mathrm{A}| \leq|\mathrm{A} \backslash \mathrm{B}|$

$\Rightarrow \mathrm{A} \preccurlyeq \mathrm{B}$ and not $\mathrm{B} \preccurlyeq \mathrm{A}$.

Proposition 9: ' $\preccurlyeq$ ' is regular'.

Proof: This is just a special case of containment', with $\mathrm{A}=\varnothing$.

Proposition 10: ‘ $\preccurlyeq$ ' satisfies complementarity.

Proof: This holds because $\mathrm{A} \backslash \mathrm{B}=\mathrm{B}^{\mathrm{C}} \backslash \mathrm{A}^{\mathrm{C}}$.

Explicitly, $\mathrm{A} \preccurlyeq \mathrm{B} \Leftrightarrow \mathrm{A} \backslash \mathrm{B}$ is finite and $|\mathrm{A} \backslash \mathrm{B}| \leq|\mathrm{B} \backslash \mathrm{A}|$

$$
\begin{aligned}
& \Leftrightarrow \mathrm{B}^{\mathrm{C}} \backslash \mathrm{A}^{\mathrm{C}} \text { is finite and }\left|\mathrm{B}^{\mathrm{C}} \backslash \mathrm{A}^{\mathrm{C}}\right| \leq\left|\mathrm{A}^{\mathrm{C}} \backslash \mathrm{B}^{\mathrm{C}}\right| \\
& \Leftrightarrow \mathrm{B}^{\mathrm{C}} \preccurlyeq \mathrm{A}^{\mathrm{C}} \text {. }
\end{aligned}
$$

Proposition 11: Suppose that a relation ' $\preccurlyeq$ ' on the subsets of a countably infinite $\Omega$ satisfies complementarity and infinity dominance. Then it is not monotonically continuous.

Proof: Let $l$ be a labeling of $\Omega$. For $i \in \mathbf{N}$, let $\mathrm{A}_{i}=\{l(0), l(1), l(2), \ldots, l(i)\}$. Let $\mathrm{B}=\{l(2)$, $l(4), l(6), \ldots\}$. For each $i, \mathrm{~A}_{i} \preccurlyeq \mathrm{B}$ by infinity dominance. But $\mathrm{U}_{i} \mathrm{~A}_{i}=\Omega$, so $\left(\mathrm{U}_{i} \mathrm{~A}_{i}\right)^{\mathrm{C}}=\varnothing$, and $\mathrm{B}$ is co-infinite. So by infinity dominance, $\left(\mathrm{U}_{i} \mathrm{~A}_{i}\right)^{\mathrm{C}} \prec \mathrm{B}^{\mathrm{C}}$, and hence it is not the case that 
$\mathrm{B}^{\mathrm{C}} \preccurlyeq\left(\mathrm{U}_{i} \mathrm{~A}_{i}\right)^{\mathrm{C}}$. By complementarity, it is not the case that $\mathrm{U}_{i} \mathrm{~A}_{i} \preccurlyeq \mathrm{B}$, so monotone continuity fails.

Lemma 2: Suppose ' $\preccurlyeq$ ' satisfies label independence' and comparability and $p: \Omega \rightarrow \Omega$ is a permutation such that $p \mathrm{~A}=\mathrm{B}$ and $p \mathrm{~B}=\mathrm{A}$. Then (i) $\mathrm{A} \sim \mathrm{B}$ and (ii) $\mathrm{A}^{\mathrm{C}} \sim \mathrm{B}^{\mathrm{C}}$.

Proof: By comparability, $\mathrm{A} \preccurlyeq \mathrm{B}$ or $\mathrm{B} \preccurlyeq \mathrm{A}$. Without loss of generality, assume $\mathrm{A} \preccurlyeq \mathrm{B}$. By Lemma $1, \mathrm{~B}=p \mathrm{~A} \preccurlyeq p \mathrm{~B}=\mathrm{A}$. This proves (i).

Complementarity would then imply (ii), but we need not assume complementarity here. Instead, observe that, since $p \mathrm{~A}=\mathrm{B}, p\left(\mathrm{~A}^{\mathrm{C}}\right)=\mathrm{B}^{\mathrm{C}}$. Similarly, $p \mathrm{~B}^{\mathrm{C}}=\mathrm{A}^{\mathrm{C}}$, so by $(\mathrm{i}), \mathrm{A}^{\mathrm{C}}=$ $\mathrm{B}^{\mathrm{C}}$

Proposition 12: If $\Omega$ is infinite and a relation ' $\preccurlyeq$ ' on the subsets of $\Omega$ satisfies transitivity, label independence', additivity', and comparability, then $\mathrm{A} \preccurlyeq \mathrm{B}$ for all $\mathrm{A}, \mathrm{B} \subseteq \Omega$.

Proof: We first show that all co-infinite sets are equivalent, and then that all infinite sets are equivalent. Since all sets are either infinite or co-infinite, and some are both, it follows by transitivity that all sets are equivalent.

Case 1: A, B are co-infinite but $\mathrm{A} \cup \mathrm{B}$ is co-finite. Then both $\mathrm{A} \backslash \mathrm{B}$ and $\mathrm{B} \backslash \mathrm{A}$ are infinite and co-infinite. Hence there is an infinite, co-infinite $\mathrm{C} \subseteq \mathrm{A} \backslash \mathrm{B}$ and a permutation $p$ such that $p \mathrm{~B}=\mathrm{C}$ and $p \mathrm{C}=\mathrm{B}$. By Lemma 2, $\mathrm{B} \sim \mathrm{C}$ and $\mathrm{B}^{\mathrm{C}} \sim \mathrm{C}^{\mathrm{C}}$. Similarly, there is an infinite, coinfinite $\mathrm{D} \subseteq \mathrm{B} \backslash \mathrm{A}$ such that $\mathrm{A} \sim \mathrm{D}$ and $\mathrm{A}^{\mathrm{C}} \sim \mathrm{D}^{\mathrm{C}}$. But $\mathrm{C}$ and $\mathrm{D}$ are disjoint, infinite, coinfinite sets, so there is a permutation $q$ such that $q \mathrm{C}=\mathrm{D}$ and $q \mathrm{D}=\mathrm{C}$. By Lemma 2 again, $\mathrm{C}$ $\sim \mathrm{D}$ and $\mathrm{C}^{\mathrm{C}} \sim \mathrm{D}^{\mathrm{C}}$. By transitivity, $\mathrm{A} \sim \mathrm{B}$ and $\mathrm{A}^{\mathrm{C}} \sim \mathrm{B}^{\mathrm{C}}$. (The equivalence of the complements will come in handy later.) 
Case 2: $\mathrm{A} \cup \mathrm{B}$ is co-infinite. Choose an infinite $\mathrm{C} \subseteq(\mathrm{A} \cup \mathrm{B})^{\mathrm{C}}$ such that $\mathrm{C}$ and $(\mathrm{A} \cup \mathrm{B} \cup \mathrm{C})^{\mathrm{C}}$ are infinite. Let $\mathrm{D}=(\mathrm{A} \cup \mathrm{B} \cup \mathrm{C})^{\mathrm{C}}$. Then $\mathrm{A} \cup \mathrm{C}$ and $\mathrm{D}$ are disjoint, infinite, co-infinite sets, so there is a permutation $p: \Omega \rightarrow \Omega$ such that $p(\mathrm{~A} \cup \mathrm{C})=\mathrm{D}$ and $p \mathrm{D}=\mathrm{A} \cup \mathrm{C}$. By Lemma 2, $\mathrm{A} \cup \mathrm{C} \sim \mathrm{D}$ and $(\mathrm{A} \cup \mathrm{C})^{\mathrm{C}} \sim \mathrm{D}^{\mathrm{C}}$. Likewise, $\mathrm{B} \cup \mathrm{C} \sim \mathrm{D}$ and $(\mathrm{B} \cup \mathrm{C})^{\mathrm{C}} \sim \mathrm{D}^{\mathrm{C}}$. Hence $\mathrm{A} \cup \mathrm{C} \sim \mathrm{B} \cup \mathrm{C}$ and $(\mathrm{A} \cup \mathrm{C})^{\mathrm{C}} \sim(\mathrm{B} \cup \mathrm{C})^{\mathrm{C}}$. (Again, the result about complements will be used later.) By additivity', A $\sim$ B.

This shows that all co-infinite sets are equivalent under ' $\preccurlyeq$ '. Now we turn to infinite sets.

Case 3: $\mathrm{A}, \mathrm{B}$ are infinite but $\mathrm{A} \cap \mathrm{B}$ is finite. Then $\mathrm{A}^{\mathrm{C}}, \mathrm{B}^{\mathrm{C}}$ are co-infinite and $\mathrm{A}^{\mathrm{C}} \cup \mathrm{B}^{\mathrm{C}}=$ $(\mathrm{A} \cap \mathrm{B})^{\mathrm{C}}$ is co-finite. Hence $\mathrm{A}^{\mathrm{C}}$ and $\mathrm{B}^{\mathrm{C}}$ satisfy the conditions of Case 1 , so $\mathrm{A}^{\mathrm{C}} \sim \mathrm{B}^{\mathrm{C}}$ and $\mathrm{A} \sim \mathrm{B}$.

Case 4: $\mathrm{A} \cap \mathrm{B}$ is infinite. Then $\mathrm{A}^{\mathrm{C}} \cup \mathrm{B}^{\mathrm{C}}$ is co-infinite, satisfying the conditions of Case 2 . As we did there, choose an infinite $\mathrm{C} \subseteq \mathrm{A} \cap \mathrm{B}$ such that $(\mathrm{A} \cap \mathrm{B}) \backslash \mathrm{C}$ is infinite. As before, we get $\left(\mathrm{A}^{\mathrm{C}} \cup \mathrm{C}\right)^{\mathrm{C}} \sim\left(\mathrm{B}^{\mathrm{C}} \cup \mathrm{C}\right)^{\mathrm{C}}$, i.e., $\mathrm{A} \backslash \mathrm{C} \sim \mathrm{B} \backslash \mathrm{C}$. By additivity, $\mathrm{A}=(\mathrm{A} \backslash \mathrm{C}) \cup \mathrm{C} \sim(\mathrm{B} \backslash \mathrm{C})$ $\cup \mathrm{C}=\mathrm{B}$.

Hence, all infinite sets are equivalent and all co-infinite sets are equivalent, so by transitivity, all sets are equivalent, or in other words, $\mathrm{A} \preccurlyeq \mathrm{B}$ for all $\mathrm{A}, \mathrm{B} \subseteq \Omega$. 


\section{References}

Benci, V., L. Horsten, and S. Wenmackers. (2013) "Non-Archimedean probability." Milan Journal of Mathematics, 81, 121-151.

----. (2018) “Infinitesimal probabilities.” British Journal for the Philosophy of Science, 69, $509-552$.

De Finetti, B. (1964) "Foresight: Its logical laws, its subjective sources." In Studies in Subjective Probability, eds. H. Kyburg and H. Smokler, 93-158. Huntington, NY: Krieger.

DiBella, N. (2018) "The qualitative paradox of non-conglomerability." Synthese, 195, 11811210. https://doi.org/10.1007/s11229-016-1261-3

Fishburn, P. C. (1986) "The axioms of subjective probability.” Statistical Science, 1, 335345. https://projecteuclid.org/euclid.ss/1177013611

Koopman, B. O. (1940) "The axioms and algebra of intuitive probability." Annals of Mathematics, 41, 269-292.

Kraft, C. H., J. W. Pratt, and A. Seidenberg. (1959) "Intuitive probability on finite sets." Annals of Mathematical Statistics, 30, 408-419.

Lewis, D. (1968) "Counterpart theory and quantified modal logic." The Journal of Philosophy, 65, 113-126.

Norton, J. D. (2018) “Eternal inflation: When probabilities fail.” Synthese. https://doi.org/10.1007/s11229-018-1734-7

-----. Manuscript. The Material Theory of Induction. http://www.pitt.edu/ jdnorton/homepage/cv.html\#material theory

Vilenkin, A. (1995) "Predictions from quantum cosmology." Physical Review Letters, 74, 846-849.

-----. (2011) “The principle of mediocrity.” Astronomy and Geophysics, 52, 5.33-5.36.

Villegas, C. (1964) “On qualitative probability a-algebras.” Annals of Mathematical Statistics, 35, 1787-1796.

Wenmackers, S., and L. Horsten. (2013) “Fair infinite lotteries.” Synthese, 190, 37-61. 